\title{
The Usefulness Of Narrow Band Imaging Endoscopy For The Real Time Characterization Of Colonic Lesions
}

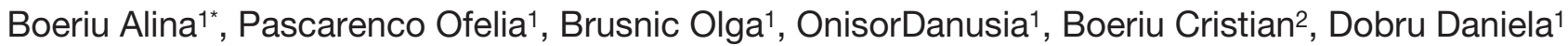 \\ 1 Department of Gastroenterology, University of Medicine and Pharmacy Tirgu Mures, Romania \\ 2 University of Medicine and Pharmacy Tirgu Mures, Tirgu Mures County Emergency Hospital, Romania
}

Narrow band imaging represents a promising endoscopic technique which allows real time characterization of colonic lesions by assessment of mucosal and vascular patterns. Due to the ability to make an optical biopsy, NBI could become a useful tool in clinical decisions regarding therapy and surveillance. The aim of this paper is to review the current knowledge on the optical diagnosis of colonic lesions by using NBI endoscopy. We reviewed research articles, reviews and meta-analyses from the PubMed and MEDLINE containing relevant data in this field. The validity of endoscopic criteria for the differentiation of adenoma and hyperplastic polyps, as well as of invasive cancer, has been evaluated in recent studies. Based on the prediction of polyp histology, new potential cost saving strategies have been proposed. Many reports are focused on the accuracy of optical biopsy by using NBI for predicting colonoscopy surveillance intervals and adenomatous histology in diminutive polyps, according to the Preservation and Incorporation of Valuable Endoscopic Innovations statement. Optical diagnosis is still under evaluation, and cannot yet be endorsed in routine practice. The high level of performance achieved by expert endoscopists needs to be replicated in the community utilizing reliable and standardized endoscopic criteria.

Keywords: narrow band imaging endoscopy, colonic polyps, optical biopsy

Received: 20 November 2015 / Accepted: 05 January 2016

\section{Introduction}

The development of advanced endoscopic techniques such as narrow band imaging endoscopy, with or without magnification, represents an important step in the improvement of characterization of colonic lesions by predicting underlying histology. This creates the possibility to adopt new strategies regarding therapy and surveillance in clinical practice. The potential benefits of the real time estimation of a polyp's histology during NBI endoscopy, so called "optical biopsy", are related to potentially decreasing total costs of colonoscopy by eliminating unnecessary biopsies and polypectomies. Recent reports have shown promising results in this field, but controversies remain. Concerns center upon a lack of standardization among different classification systems, variable inter-observer agreement, and lagging clinical acceptance.

The aim of this paper is to assess the current knowledge on the optical diagnosis of colonic lesions by using NBI endoscopy. We reviewed research articles, reviews and meta-analyses published in the last eight years in the PubMed and MEDLINE databases for the following search terms: narrow band imaging endoscopy, colonic polyps, and colorectal neoplasia.

\section{Principles of the method}

Narrow band imaging (NBI) represents a method of highresolution endoscopic imaging which allows a detailed evaluation of surface patterns. The basic principle of the

* Correspondence to: Alina Boeriu

E-mail: aboeriu@gmail.com method consists in the use of lights with narrowed bandwidths that penetrate superficial layers of the intestinal wall and are easily absorbed by hemoglobin. Thus, optical filters select blue $(415 \mathrm{~nm})$ and green light $(540 \mathrm{~nm})$, allowing the visualization of capillaries in the superficial mucosal layer (brown aspect) and deeper mucosal and submucosal vessels (cyan appearance), while the red light with deeper penetration in the tissues is taken out. This leads to an enhancement of mucosal and vascular details. The assessment of vascular and mucosal morphologic changes facilitates the histological prediction during the NBI examination (optical biopsy) and the real time distinction between non-neoplastic and neoplastic colonic lesions. As in all new technologies, there is a learning curve in accurately identifying and differentiating normal and modified patterns.

\section{Characterization of colonic lesions}

Different classification systems based on NBI assessment of colorectal polyps with or without magnification have been described in medical reports, as well as their usefulness in the real time estimation of polyp histology. A proper characterization of lesions consists in the evaluation of mucosal patterns and vascular architecture. Distinct mucosal patterns have been detected through magnified examination of colonic polyps. Kudo et al. have described a classification system of mucosal pit pattern that included 5 types of pits: type I- round pits, type II- stellar or papillary pits, type III L- large tubular or roundish pits, type III s- small tubular or roundish pits, type IV- branch-like or gyrus-like pits and type V-non-structural pits [1]. The assessment of vascular patterns represents a reliable method for differ- 
entiating non-neoplastic from neoplastic colorectal polyps. Sano et al. have proposed the "meshed capillary" (MC) vessels classification for differentiating colonic lesions. Lesions with invisible or faintly visible MC vessels under NBI with magnification are hyperplastic polyps (type I pattern), whereas lesions with clearly visible MC vessels (type II pattern) are neoplastic (adenomatous polyps). MC vessels are disorganized in cancerous lesions (type III pattern) [2]. The MC pattern evaluation represents a useful diagnostic tool in differentiating small colonic polyps (diagnostic accuracy, sensitivity, and specificity $95.3 \%, 96.4 \%$, and $92.3 \%$, respectively) [3].

By combining of mucosal (Kudo's pit pattern) and vascular criteria (Sano classification), distinct endoscopic features have been described that correspond with different types of colonic lesions. Singh et al. demonstrated that the combined NBI classification could be successfully used in routine clinical practice to predict histology with higher accuracy than high-resolution white light endoscopy (WLE). The type I or II Kudo's mucosal pattern (round, stellar or papillary pits) and type I vascular pattern (absence of vascular structure) were detected in hyperplastic polyps (Fig. 1 ); type III or type IV Kudo's mucosal pattern (tubular or branching pits) and type II vascular pattern (regular vessels) were detected in adenoma (Fig. 2); type V mucosal pattern (disappeared pits) and type III vascular pattern (irregular vessels) correspond to adenocarcinoma [4].

Another classification system including vascular and surface pattern observation by NBI magnification endoscopy has been proposed by Kanao et al. In type A lesions, microvessels were not observed or were extremely opaque; in type B lesions fine microvessels were detected surround the pits; in type $\mathrm{C}$ irregular microvessels with heterogenous diameter or distribution were observed. Type A has been mainly detected in hyperplastic polyps, type B in adenoma and intramucosal cancer. Type $\mathrm{C}$ has been found in adenomas and carcinomas. Type $\mathrm{C}$ is divided in 3 subtypes

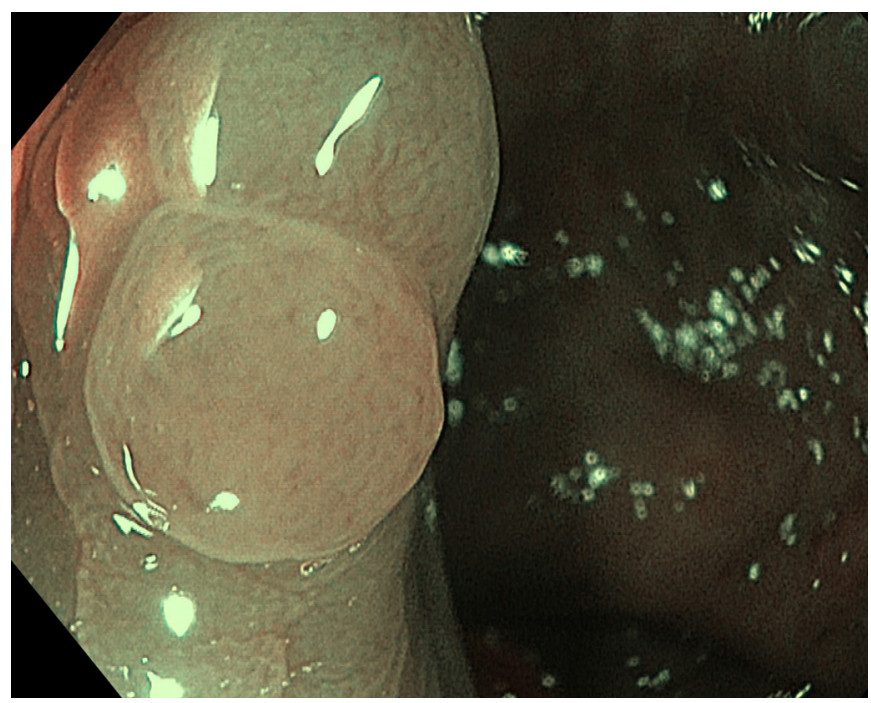

Fig.1. Hyperplastic polyps: pale, bland color, lacy or no vessels
(C1, C2, C3), according to pit visibility, vessel diameter, irregularity, and distribution, in the attempt to assess invasion depth of cancer. In type $\mathrm{C} 1$ (Fig. 3), detected in adenoma and in intramucosal cancer as well, vessel diameter or distribution is homogenous, while in type C3, mainly detected in massively invaded submucosal cancer, irregular vessel diameter is thick, vessel distribution is heterogenous, and avascular areas are observed (Fig. 4). However, a clear estimation of submucosal invasion, and subsequent therapeutic strategy, could not be determined on the basis of this classification, as type C2 (microvessels comprising irregular network, irregular surface patterns, heterogenous vessel diameter or distribution- Fig. 5) has been detected in both intramucosal cancer as well as in massively invaded submucosal cancer [5].

More recently, an international expert group for the diagnosis of colonic lesions has proposed the NBI International Colorectal Endoscopic (NICE) classification, based upon the evaluation of lesion color, vascularization and surface pattern [6]. This classification is suitable for NBI endoscopic evaluation, with or without use of magnification. Three types of colonic lesions are included, with their specific mucosal and vascular features. The endoscopic features for identifying hyperplastic polyps (Type 1 lesions) are: the similar or lighter color of the polyp compared with the background mucosa, with no vessels or isolated vessels coursing across the polyp surface; the homogenous lack of mucosal pattern or the detection of dark or white spots of uniform size (Fig. 1). For the Type 2 lesions (adenomas with low or high- grade dysplasia, and adenomas with superficial submucosal carcinoma), the following endoscopic criteria should be assessed: the browner color of the lesion relative to the background mucosa; the visualization of brown vessels surrounding oval, tubular or branched white structures (Fig. 2). According to NICE classification, deep submucosal invasive cancer (Type 3 lesion) shows particular mucosal and vascular features (Fig. 4): a brown color of

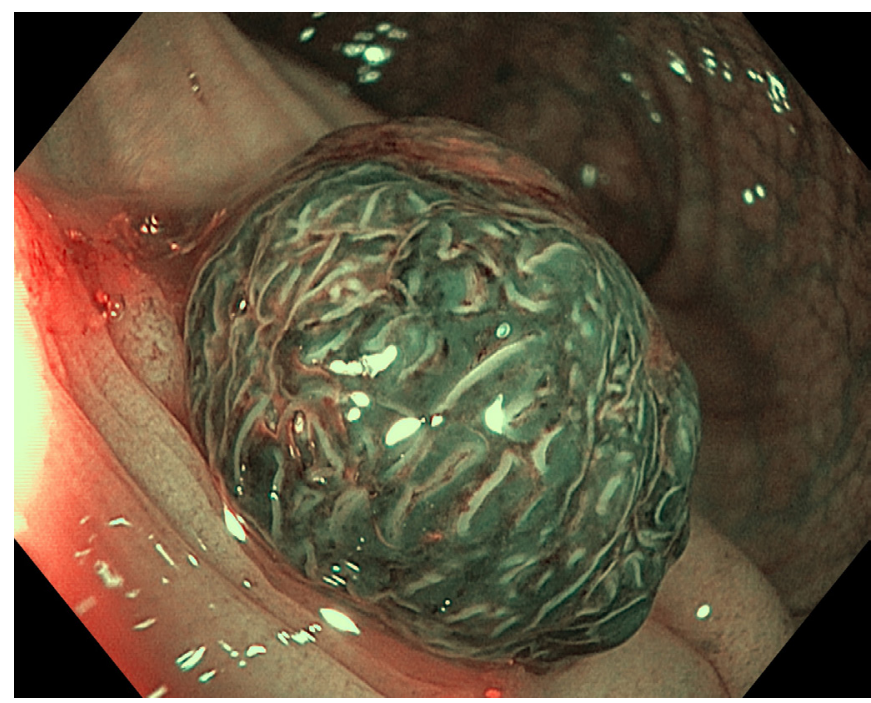

Fig.2. Adenomatous polyp: brown color, thick brown vessels surrounding tubular pits 


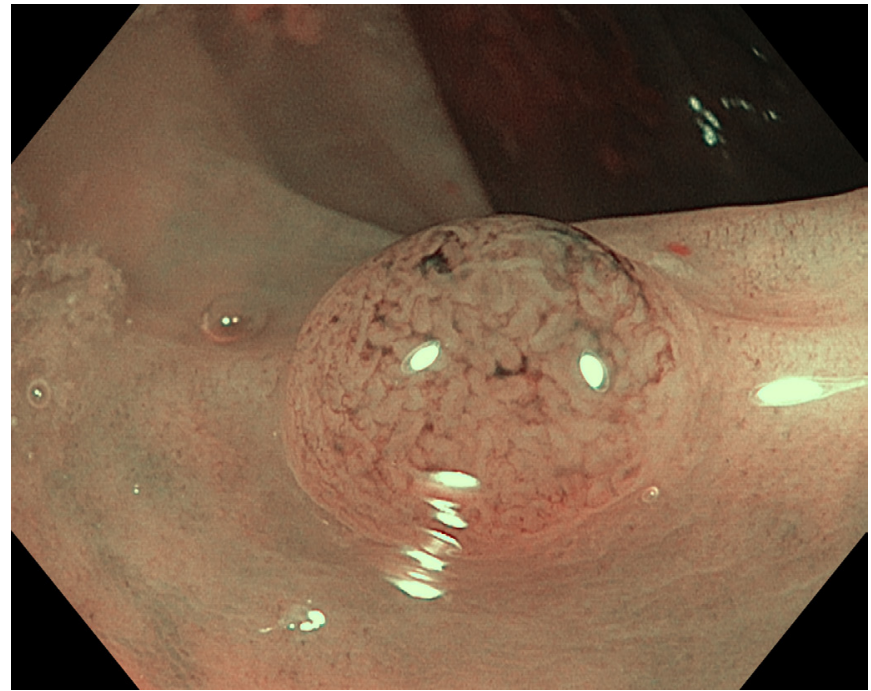

Fig.3. Low-grade adenoma: vessel diameter or distribution is homogenous

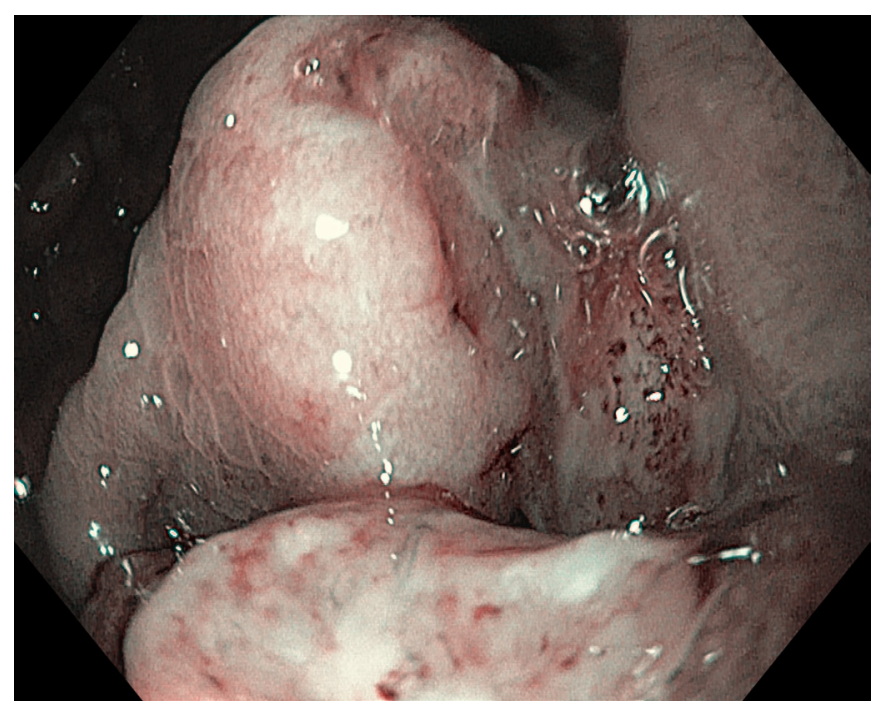

Fig.4. Invasive submucosal cancer: vessel distribution is heterogenous, avascular areas are detected

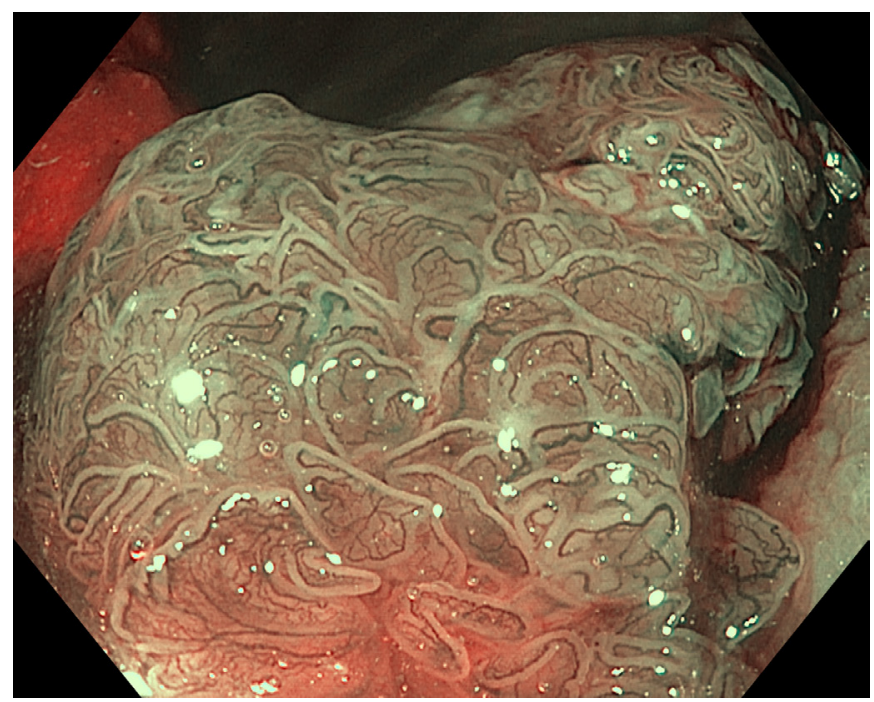

Fig.5. High-grade adenoma: irregular vascular network, vessel diameter or distribution is heterogenous the lesion relative to background, sometimes with patchy whiter areas; an absent or amorphous mucosal pattern; a vascular pattern with disrupted or missing vessels [7].

The validity of NICE classification system in differentiating hyperplastic versus adenomatous polyps histology by using NBI without magnification has been reported $(89 \%$ accuracy, 98\% sensitivity, 95\% NPV) [6]. Therefore, optical diagnosis of colorectal polyps according to NICE criteria has been proposed as a tool to guide therapeutic decisions and surveillance strategies in clinical practice.

In summary, the main contribution of NBI technique consists in the differentiation of colonic lesions. Regarding the detection of lesions, it has been shown that conventional colonoscopies are associated with miss rates for polyps, especially for sessile and flat lesions [8]. For this reason, new technologies have been developed in order to optimize adenoma detection. The ability of NBI endoscopy to improve the detection of polyps has been evaluated in different studies, and contradictory results have been obtained. Some authors have reported an increase in adenoma detection rate, as well as in diminutive adenoma detection rate by performing NBI colonoscopy, rather than conventional colonoscopy [9].

Other studies have shown no improvement in adenoma detection rate using NBI endoscopy versus white light endoscopy. Even so, a lower miss rate for adenoma in the proximal colon was achieved by performing NBI colonoscopy [10], while the detection rate of flat adenomas has increased with NBI [11]. Recent meta-analyses and systematic reviews have also shown that NBI provides no increase in adenoma or polyp detection rates compared with conventional colonoscopy $[12,13]$. However, after exclusion of high-definition television modalities, the rate of adenomas detection by NBI compared with white light was significantly higher, especially in patients with one adenoma. The detection of flat adenoma has also increased using NBI colonoscopy [13]. Due to conflicting data from published trials, the potential benefit of NBI technique in neoplastic colonic lesions detection, especially in right-sided lesions and flat adenoma, requires further evaluation.

\section{Clinical strategies and controversies}

NBI with or without association of magnifying endoscopy has proved to improve the prediction of polyp histology during the real time endoscopy. On this basis, two different strategies for the management of diminutive colorectal polyps ( $\leq 5 \mathrm{~mm}$ in size) have been proposed. The "resect and discard" strategy represents an option for the management of diminutive and small adenomatous polyps (less than $10 \mathrm{~mm}$ in size), which means the real time recognition of histology by NBI evaluation, followed by resection and discard of polyps without sending for histologic assessment [14]. The post-polypectomy surveillance intervals might be predicted on the basis of estimated histology of diminutive polyps by NBI and the histopathologic assessment of larger polyps [15]. 
In case of diminutive rectosigmoidian hyperplastic polyps, the "do not resect" strategy could be applied in practice. These polyps without malignant potential could be left in place on the basis of optical diagnosis by NBI, without resection or histologic analysis [16]. The potential benefit of these strategies consists in decreasing the global cost of colonoscopy [17], as well as in decreasing possible complications related to polypectomy. For right-sided lesions, a resection and surveillance strategy is advocated due to their malignant potential [18].

According to the recommendations of The American Society for Gastrointestinal Endoscopy, an endoscopic technique could be used for characterization of colonic polyps, and for guiding the "resect and discard" strategy, only if achieves the criteria endorsed by the Preservation and Incorporation of Valuable Endoscopic Innovations (PIVI) statement. The thresholds for an accurate assessment of a polyp's histology are: the technology should provide $\geq 90 \%$ negative predictive value (when used with high confidence) for adenomatous histology in diminutive rectosigmoid polyps; optical diagnosis for diminutive polyps combined with pathologic assessment of polyps larger than $5 \mathrm{~mm}$ should provide $\geq 90 \%$ agreement in assignment of post-polipectomy surveillance intervals when compared with decisions based on histopathological assessment of all identified polyps [19]. Recent reports have tested the clinical applicability of optical diagnosis by NBI endoscopy and have investigated the ability of the method to reach the PIVI criteria $[20,21]$.

There are some issues related to optical biopsy of colorectal polyps. One is the challenge presented by the real time diagnosis of serrated polyps. The distinction between sessile serrated adenomas (SSAs) and hyperplastic polyps is difficult during conventional colonoscopy. Still, the accurate diagnosis of SSAs is mandatory for colorectal cancer prevention since these lesions harbor malignant potential and are associated with an increased risk of synchronous cancer $[22,23]$. Reliable NBI criteria for the differentiation between hyperplastic polyps and SSAs have not yet been defined. In fact, recent reports from community gastroenterologists have detected among SSAs intermediate features between hyperplastic polyps and adenomas according to NICE classification [24].

The accurate interpretation of mucosal and vascular patterns represents another issue related to the incorporation of the optical diagnosis in routine practice. Most studies have been conducted in academic centers and have been performed by experts. They have obtained a good diagnostic accuracy for real time assessment of colonic polyps. Repici et al. have recently reported a high confidence prediction of histology in diminutive polyps ( $92 \% \mathrm{NPV})$, when NBI colonoscopies have been performed by experienced endoscopists [21].

Other studies have reported different levels of accuracy for the technique in community practice. Ladabaum et al. have found less accurate results in the community setting.
The thresholds for optical biopsy in community practice did not entirely meet PIVI criteria. (20). Training programs including ex vivo evaluation of photographs of colonic polyps with known histology, real-time optical diagnosis of polyps during NBI colonoscopy, and comparison with histopathologic results, as well as computerized training modules, represent the methods proposed to improve diagnostic accuracy $[25,26]$.

\section{Conclusion}

Endoscopic assessment of mucosal and vascular features by NBI is useful for real time prediction of polyp histology. The advantages of the method in the characterization and differentiation of colonic lesions have been emphasized in many reports. Characteristic endoscopic features associated with hyperplastic and adenomatous polyps have been described, as well as for invasive cancer. The performance of several classification systems has been evaluated in different studies. The real time detection of SSAs still represents a challenge, since accurate NBI criteria for the differentiation between SSAs and hyperplastic polyps are missing.

NBI endoscopy has proven to contribute to the differentiation between non-neoplastic and neoplastic polyps, but conflicting data regarding the improvement of colorectal polyp detection have been reported. Accurate prediction of histology during endoscopy could be advantageous in promoting real-time decisions regarding therapy and surveillance. According to ASGE recommendations, minimum thresholds should be reached in order to adopt the "characterize, resect and discard" strategy in clinical practice.

Training and expertise in accurately differentiating mucosal and vascular patterns are mandatory and some methods have been proposed for improving diagnostic performance. Large multicenter studies are needed for the validation and standardization of optical diagnosis, before its acceptance into routine practice.

\section{Acknowledgements}

This paper is supported by the Internal Research Grant CIGCS UMF TGM-PO-CC-01-F01, financed by University of Medicine and Pharmacy Tirgu Mures.

\section{Conflict of interest}

None to declare.

\section{References}

1. Kudo S, Tamura S, Nakajima T, et al. Diagnosis of colorectal tumorous lesions by magnifying endoscopy. Gastrointest Endosc 1996;44:8-14.

2. Sano Y, Horimatsu T, Fu IK, et al. Magnifying observation of microvascular architecture of colorectal lesions using a narrow band imaging system. Dig Endosc 2006;18(Suppl1):S44-S51.

3. Sano Y, Ikematsu H, Fu Kl, et al. Meshed capillary vessels by use of narrow-band imaging for differential diagnosis of small colorectal polyps. Gastrointest Endosc 2009;69:278-283.

4. Singh R, Owen V, Shonde A, et al. White light endoscopy, narrow band imaging and chromoendoscopy with magnification in diagnosing colorectal neoplasia. World J Gastrointest Endosc 2009;1(1):45-50.

5. Kanao H, Tanaka S, Oka S, et al. Narrow-band imaging magnification predicts the histology and invasion depth of colorectal tumors. 
Gastrointest Endosc 2009;69:631-636.

6. Hewett GD, Kaltenbach T, Sano Y, et al. Validation of a Simple Classification System for Endoscopic Diagnosis of Small Colorectal Polyps Using Narrow-Band Imaging. Gastroenterology 2012;143:599-607.

7. Hayashi N, Tanaka S, Hewett DG, et al. Endoscopic prediction of deep submucosal invasive carcinoma: validation of the Narrow-Band Imaging International Colorectal Endoscopic (NICE) classification. Gastrointest Endosc 2013;78:625-632.

8. Heresbach D, Barrioz T, Lapalus MG, et al. Miss rate for colorectal neoplastic polyps: a prospective multicenter study of back-to-back video colonoscopies. Endoscopy 2008;40:284-290.

9. Inoue T, Murano M, Murano T, et al. Comparative study of conventional colonoscopy and pan-colonic narrow-band imaging system in the detection of neoplastic colonic polyps: a randomized, controlled trial. J Gastroenterol 2008;43:45-50.

10. Ikematsu $H$, Saito $Y$, Tanaka $S$, et al. The impact of narrow band imaging for colon polyp detection: a multicenter randomized controlled trial by tandem colonoscopy. J Gastroenterol 2012;47:1099-1107.

11. Paggi S, Radaelli F, Amato A, et al. The impact of narrow band imaging in screening colonoscopy: a randomized controlled trial. Clin Gastroenterol Hepatol 2009;7:1049-1054.

12. Dinesen L, Chua TJ, Kaffes AJ. Meta-analysis of narrow-band imaging versus conventional colonoscopy for adenoma detection. Gastrointes Endosc 2012;75:604-611.

13. Jin XF, Chai T-H, Shi J-W, Yang X-C, Sun Q-Y. Meta-analysis for evaluating the accuracy of endoscopy with narrow band imaging in detecting colorectal adenomas. J Gastroenterol Hepatol 2012;27:882-887.

14. Ignjatovic A, East JE, Suzuki N, et al. Optical diagnosis of small colorectal polyps at routine colonoscopy (Detect InSpect ChAracterise Resect and Discard; Discard trial): a prospective cohort study. Lancet Oncology 2009:10:1171-1178.

15. Gupta N, Bansal A, Rao D, et al. Accuracy of in vivo optical diagnosis of colon polyp histology by narrow-band imaging in predicting colonoscopy surveillance intervals. Gastrointest Endosc 2012;75(3):494-502.

16. Hewett D, Huffman ME, Rex DK. Leaving distal colorectal hyperplastic polyps in place can be achieved with high accuracy by using narrow-band imaging: an observational study. Gastrointest Endosc 2012;76:374-380.

17. Hassan C, Pickhardt PJ, Rex DK. A resect and discard strategy would improve cost-effectiveness of colorectal cancer screening. Clin Gastroenterol Hepatol 2010;8:865-869.

18. Paggi S, Rondonotti E, Amato A, et al. Resect and discard strategy in clinical practice: a prospective cohort study. Endoscopy 2012;44:899904.

19. Rex DK, Kahi C, O'Brien M, et al. The American Society for Gastrointestinal Endoscopy PIVI (Preservation and Incorporation of Valuable Endoscopic Innovation) on real-time endoscopic assessment of the histology of diminutive colorectal polyps. Gastrointest Endosc 2011;73:419-422.

20. Ladabaum U, Fioritto A, Mitani A, et al. Real-Time Optical Biopsy of Colon Polyps With Narrow Band Imaging in Community Practice Does Not Meet Key Thresholds for Clinical Decisions. Gastroenterology 2013;144:81-91.

21. Repici A, Hassan C, Radaelli F, et al. Accuracy of narrow-band imaging in predicting colonoscopy surveillance intervals and histology of distal diminutive polyps: results from a multicenter, prospective trial. Gastrointest Endosc 2013;78:106-114.

22. Leggett B, Whitehall V. Role of the serrated pathway in colorectal cancer pathogenesis. Gastroenterology 2010;138:2088-2100.

23. Hiraoka S, Kato J, Fujiki S, et al. The presence of large serrated polyps increases risk for colorectal cancer. Gastroenterology 2010;139:1503,10,1510.e1-3.

24. Kumar S, Fioritto A, Mitani A, et al. Optical biopsy of sessile serrated adenomas: do these lesions resemble hyperplastic polyps under narrowband imaging? Gastrointest Endosc 2013;78(6):902-909.

25. Ignjatovic A, Thomas-Gibson S, East JE, et al. Development and validation of a training module an the use of narrow-band imaging in differentiation of small adenomas from hyperplastic colorectal polyps. Gastrointest Endosc 2011;73:128-133.

26. Patel SG, Rastogi A, Austin G, et al. Gastroenterology trainees can easily learn histologic characterization of diminutive colorectal polyps with narrow band imaging. Clin Gastroenterol Hepatol 2013;11:997-1003. 